\title{
Non-equilibrium molecular dynamics simulations of the thermal transport properties of Lennard-Jones fluids using configurational temperatures
}

\author{
Niall Jackson ${ }^{a, b},{ }^{*}$ J. Miguel Rubi ${ }^{c}$, and Fernando Bresme ${ }^{a, b, d \dagger}$ \\ ${ }^{a}$ Department of Chemistry, Imperial College London, $S W^{r} 72 A Z$, \\ London UK. ${ }^{b}$ Thomas Young Centre for Theory and Simulation of Materials, \\ Imperial College, ${ }^{c}$ Departmento de Fisica, Universidad Autónoma de Barcelona, Barcelona, \\ Spain ${ }^{d}$ Department of Chemistry, Norwegian University of Science and Technology, Trondheim, Norway
}

\begin{abstract}
We investigate the accuracy of two expressions for configurational temperature when calculating thermal transport properties in non-equilibrium systems under thermal gradients. The temperature $T_{\mathrm{conF}}$, introduced by Jepps et. al [Phys. Rev. E 624757 (2000)] is found to give results in almost exact agreement with the equipartition temperature for the thermal conductivity of the Lennard-Jones fluid, and for the temperature profile across a solid-liquid interface. Measurements of $T_{\mathrm{conF}}$ are, however, less precise than those of the equipartition temperature, which results in less accurate measurements of the interfacial thermal conductance across a liquid-vapour interface. The approximate expression $T_{\text {con1 }}$, which depends strongly on the number of sampled atoms, predicts unphysical negative thermal conductances in liquid-vapor interfaces, highlighting the limitations of some definitions of the configurational temperature in the computation of thermal transport properties via non-equilibrium simulations.
\end{abstract}

\section{INTRODUCTION}

Thermal transport plays an important role in technological and industrial applications [1]. A sound understanding of thermal transport in materials and fluids is of great importance to advance in the design of nanofluids for thermal management problems as well as in the development of thermal devices, such as thermoelectrics [2], and thermal separators. Recent realisations of the latter rely on the application of thermal gradients to fluid mixtures consisting of particles with different masses. The thermal gradient can be exploited in this instance to induce particle sorting across a nanopore [3].

Computer simulations have proven to be an ideal tool to quantify the behavior of fluids and materials under thermal gradients. It has been possible to uncover novel non-equilibrium coupling effects leading to the preferred orientation of molecular fluids $[4,5]$, and study thermodiffusion. In this way simulations have provided a better understanding of the microscopic variables determining the thermodiffusive and thermophoretic response of suspensions and molecular fluids [6-13]. An account of the applicability of non-equilibrium simulations to quantify transport properties and coupling phenomena in fluids and fluid interfaces is given in reference [14].

Although thermal transport coefficients can be computed using equilibrium simulations via the time correlation function formalism [15], non-equilibrium simulations offer definite advantages in the computation of complex coupling phenomena in mixtures. There are two main methods to perform non-equilibrium simulations under thermal gradients, the synthetic approach [16], which relies on the calculation of correlation functions under the

\footnotetext{
*Electronic address: n.jackson12@imperial.ac.uk

${ }^{\dagger}$ Electronic address: f.bresme@imperial.ac.uk
}

influence of a fictitious field, and the boundary driven non-equilibrium molecular dynamics (BD-NEMD) approach, in which a thermal gradient is explicitly simulated by inducing a heat flux along the simulation box. The flux is normally driven by adding/withdrawing energy $[17,18]$ or by directly thermostatting the molecules in predefined boundary regions [19]. The BD-NEMD approach has become a popular choice, as it is easy to implement, and no a priori knowledge of the microscopic heat flux equation (MHFE) is required, since the heat flux can be extracted from an analysis of the rate of energy exchange. The computation of the thermal gradient in BD-NEMD simulations requires the calculation of a temperature profile. This is normally done by dividing the system into small slabs perpendicular to the direction of the heat flux, and then computing the temperature locally using the equipartition principle. This approach has been tested in detail in a number of publications, and it has been demonstrated that thermodynamic and dynamic properties can be computed locally [20], a result that supports the local equilibrium hypothesis [21].

Although temperature is a well defined concept under equilibrium conditions, the measurement of temperatures in non-equilibrium systems has motivated a number of works suggesting that the measurement may be problematic $[22,23]$. The applicability of the standard thermodynamic definition of temperature, $1 / T=(\partial S / \partial U)_{V, N}$ to non-equilibrium systems has been discussed - in particular whether the entropy, $S$, is defined in non-equilibrium systems. This has prompted the search for suitable definitions (see reference [22] for a comprehensive discussion), and a discussion of whether it is even possible to define a unique temperature away from equilibrium. Recently, there has been a significant amount of activity on the computation of configurational temperatures - expressions which involve only functions of atomic coordinates, and not their momenta. Since these expressions provide an independent measurement of the tempera- 
ture, agreement between the kinetic and configurational approaches can be taken as evidence of local thermodynamic equilibrium in a non-equilibrium system.

The modern interest in configurational temperature began with the work of Rugh [24], although the concept itself follows from Yvon's theorem (see equation (7.2.11) and equation (7.2.12) in reference [25], page 186) formulated in the canonical ensemble, and also appears in the statistical mechanics monograph by Landau and Lifschitz (see reference [26], page 103). Rugh derived an equation that allows the computation of a so-called "dynamical" temperature from the divergence of the Hamiltonian. That approach holds in the microcanonical ensemble only, but Jepps et al. [27] extended the idea to the canonical ensemble, and derived equations consistent with the periodic boundary conditions employed in molecular dynamics simulations. We briefly recap these results to introduce the temperature measurements that we will use in our simulations.

The equipartition principle establishes that the temperature can be evaluated from the kinetic temperature, $T_{k i n}$. For $N$ particles,

$$
\frac{1}{k_{B} T_{\text {kin }}}=\left\langle\frac{3 N}{\sum_{i=1}^{N} \frac{\mathbf{p}_{i}^{2}}{m_{i}}}\right\rangle
$$

where $\mathbf{p}_{i}$ is the momentum of particle $i$ with mass $m_{i}$, and $k_{B}$ is the Boltzmann constant. This is the thermometer used as standard in molecular dynamics simulations.

Rugh [24] used the thermodynamic definition that relates the temperature, $T$, with the entropy, $S$ and internal energy $E$,

$$
\frac{1}{T}=\left(\frac{\partial S}{\partial E}\right)_{V, N}
$$

to derive an expression for the dynamical temperature in the microcanonical ensemble,

$$
\frac{1}{k_{B} T}=\left\langle\nabla \cdot \frac{\nabla \mathcal{H}(\Gamma)}{|\nabla \mathcal{H}|^{2}}\right\rangle
$$

where the angular brackets have the usual meaning of an ensemble average, $k_{B}$ is the Boltzmann constant, and $\mathcal{H}(\Gamma) \equiv \mathcal{H}\left(\mathbf{r}^{N}, \mathbf{p}^{N}\right)$ is the Hamiltonian of the system, which is a function of the coordinates, $\mathbf{r}^{N}$, and momenta, $\mathbf{p}^{N}$, of all $N$ particles.

Jepps et al. [27] subsequently extended this work to the canonical ensemble with periodic boundary conditions, producing their own family of temperature expressions. Their purely configurational result reads

$$
\frac{1}{k_{B} T_{\mathrm{conF}}}=-\frac{\left\langle\sum_{i} \nabla_{i} \cdot \mathbf{F}_{i}\right\rangle}{\left\langle\sum_{i=1}^{N} \mathbf{F}_{i}^{2}\right\rangle}
$$

where the divergence operator is $\nabla_{i}=\left(\frac{\partial}{\partial x_{i}}, \frac{\partial}{\partial y_{i}}, \frac{\partial}{\partial z_{i}}\right)$ and $\mathbf{F}_{i}$ is the force acting on particle $i$. The subscript con indicates that the temperature is purely configurational, and subscript $\mathrm{F}$ indicates that the temperature is a fraction of two averages. They were also able to produce a purely configurational temperature using Rugh's approach,

$$
\frac{1}{k_{B} T_{\mathrm{conR}}}=\left\langle-\frac{\sum_{i=1}^{N} \nabla_{i} \cdot \mathbf{F}_{i}}{\sum_{i=1}^{N} \mathbf{F}_{i}^{2}}-\frac{2 \sum_{i \neq j}^{N} \mathbf{F}_{i} \mathbf{F}_{j}: \nabla_{i} \mathbf{F}_{j}}{\left(\sum_{i=1}^{N} \mathbf{F}_{i}^{2}\right)^{2}}\right\rangle
$$

where the subscript $\mathrm{R}$ indicates that the temperature arises from Rugh's method. The second term of this equation decays as $\mathcal{O}(1 / N)$ and can therefore be neglected in the thermodynamic limit, leading to an approximate expression,

$$
\frac{1}{k_{B} T_{\operatorname{con} 1}}=-\left\langle\frac{\sum_{i=1}^{N} \nabla_{i} \cdot \mathbf{F}_{i}}{\sum_{i=1}^{N} \mathbf{F}_{i}^{2}}\right\rangle
$$

The subscript 1 indicates that the expression is arrived at by taking only the first term of equation (5). This is an attractive prospect, as calculating the tensor product is computationally expensive. Although the resulting equation is superficially similar to equation (4), however, neglecting the second term causes $T_{\text {con1 }}$ to vary much more strongly with density than either $T_{\text {conf }}$ or $T_{\text {kin }}[27,28]$.

This formulation of the configurational temperature has been employed to test the temperature generated in canonical Monte-Carlo simulations [29], in experiments of confined colloids as a probe of colloid-surface interactions [30], and to develop reverse mapping algorithms to extract pair potentials [31]. It has also become possible to measure configurational temperatures in systems with discontinuous potentials [28, 32], and to employ thermostats that act only on the configurational degrees of freedom [33]; while it is well established that linear transport coefficients (such as viscosity and thermal conductivity) do not depend significantly on the choice of thermostat, it has been shown that nonlinear properties, such as normal stress coefficients, can do [34]. There is no reason a priori that any one temperature should be a better choice than others; in fact, Ayton et al. [35] established that in systems experiencing spatially varying strain rates, only Rugh's dynamical temperature is able to correctly account for the resulting heat fluxes. Several articles have reported configurational temperatures in non-equilibrium systems under shear where the temperature can feature spatial anisotropy, and differences between the temperatures obtained from the kinetic and configurational routes have been reported in models of polymer fluids [36]. In a recent investigation of argon confined in zeolite structures [37], it was found that the kinetic and configurational approaches can yield significantly different results. These results disagree with previous studies performed in equilibrium systems, where good agreement between both temperature definitions, 
kinetic and configurational, is generally found [27]. The comparison of kinetic and configurational temperatures in steady state systems under thermal gradients has received less attention, but it has been noted that the color conductivity algorithms result in anisotropic configurational temperatures which differ from the kinetic ones [38]. Recently the impact of the potential cutoff on the calculation of configurational temperatures proposed by Jepps et al. [27] has been considered [28], including the computation of systems under temperature gradients. It was found that for sufficiently long cutoff distances $T_{\text {conF }}$ is in excellent agreement with the kinetic temperature.

All of these works are interesting, and they raise some conceptual questions regarding the thermometer used to monitor the temperature of a system, as well as more practical questions regarding the actual computation of the temperature in non-equilibrium systems. Previous works have found that the configurational and kinetic temperatures are consistent at equilibrium conditions, although large differences have also been reported when the temperatures are computed locally by dividing the system in small sub-volumes. Non-equilibrium simulations of fluid and suspensions under thermal gradients require the computation of the local temperatures in small sub-volumes, which are used to obtain thermal gradients and thermal conductivities. In this article we investigate the applicability of $T_{\text {conF }}$ to the computation of transport properties, thermal conductivity and thermal conductance, of fluids and solids under thermal gradients, and compare to the superficially similar $T_{\text {con1 }}$.

Our paper is structured as follows: we begin with a discussion of the non-equilibrium computer simulation strategy and the simulated force-fields, before discussing our main results for bulk and interfacial systems under thermal gradients, and finally we present our main conclusions and final remarks.

\section{METHODOLOGY}

All our our simulations were performed using the truncated and shifted Lennard-Jones potential,

$$
U(r)= \begin{cases}4 \varepsilon\left[\left(\frac{\sigma}{r}\right)^{12}-\left(\frac{\sigma}{r}\right)^{6}\right]-U\left(r_{c}\right) & r<r_{c} \\ 0 & r \geq r_{c}\end{cases}
$$

where $\varepsilon$ represents the interaction strength, $\sigma$ the particle diameter and $r_{c}$ the potential cutoff, which was set in all our simulations to $r_{c}=5 \sigma$. We chose this comparatively long cutoff to avoid potential problems associated with the truncation of the potential, and discontinuities in its derivatives. We demonstrate in appendix A how the various temperature expressions, equations (4) and (6), can be evaluated with this potential. Unless otherwise stated, we work throughout in Lennard-Jones reduced units.

In addition to control simulations in the microcanonical (NVE) ensemble, we performed non-equilibrium molecular dynamics simulations of bulk and interfacial systems under thermal gradients. We employed boundary driven simulations in prismatic boxes with aspect ratio $L_{x}=L_{y}$, with $L_{z}$ varied to control the bulk density. Two regions inside the simulation box were defined, one at the center of the simulation cell and two at the edges (see, for example, figure 9). These regions were thermostatted at the desired temperatures every timestep, followed by a reset of the total momentum of the simulation box. In this work we employed a simple velocity rescaling approach, where the new velocity of particle $i$, $v_{i, \text { new }}=\chi v_{i, \text { old }}$, is given by the velocity before rescaling $\left(v_{i, \text { old }}\right)$ and the rescaling constant, $\chi=\sqrt{K_{\mathrm{t}} / K_{\text {old }}}$, which is defined by the ratio of the old and target kinetic energies. This implementation is similar to the thermostatting BD-NEMD scheme [19].

Once the stationary state is established, the thermal gradient can be computed from the derivative of the temperature profile. The latter is obtained by dividing the simulation box into layers, typically 100 in this work, perpendicular to the direction of the gradient, and by computing the temperature locally in each layer. Local values of $T_{\text {kin }}, T_{\text {conF }}$, and $T_{\text {con1 }}$ were computed for all of our NEMD simulations. To calculate the configurational temperatures, we included all the neighbour particles inside the cutoff radius from a given particle, irrespective of whether the neighbors were in the same layer occupied by the particle.

The heat flux at stationary conditions was obtained from the rate of energy exchange at the hot and cold thermostats, $J_{Q}=Q /(2 A \delta t)$, where $Q$ is the amount of kinetic energy exchanged per timestep, $\delta t$, across the thermostat cross-sectional area, $A=L_{x} \times L_{y}$. The factor of 2 accounts for the two heat fluxes present in the simulation box, which are equal by symmetry.

All the simulations were performed in parallel with LAMMPS [39], using a reduced timestep of $\delta t=0.0025$. In the equilibrium simulations, the production run of $2 \times 10^{6}$ steps was preceded by an equilibration period of $10^{5}$ steps. In simulations involving temperature gradients, the systems were equilibrated for a period of $10^{5}$ steps before the thermostats were switched on. This was followed by another equilibration period of $10^{5}$ steps to establish the non-equilibrium steady state, and $2 \times 10^{7}$ steps for the production run. During production, the positions and velocities of all atoms were recorded every 1000th time step. Results were averaged over three independent simulations, with different initial velocities, to produce the final temperatures. A custom postprocessing code was used to calculate all the temperatures discussed above using the method outlined in appendix A. 


\section{RESULTS}

\section{A. Bulk systems}

Although $T_{\text {con1 }}$ has a strong density dependence, and agrees poorly with $T_{\text {kin }}$ at low densities, at typical liquid densities $(\rho \sim 0.7)$ the discrepancy is rather small $(<1 \%)[27$. Lervik et al. [28] showed that the average value $T_{\text {conf }}$ does not change when it is evaluated in subvolumes, and so is appropriate for use in non-equilibrium simulations. We begin by considering $T_{\text {con } 1}$ in the same situation. Equilibrium simulations were performed according to the procedure described in section II, with $N=8000$ atoms at $T=1.5$ and $\rho=0.7$. This supercritical temperature was chosen to avoid problems connected to the vicinity of the critical point. All temperature measurements were taken in the NVE ensemble.
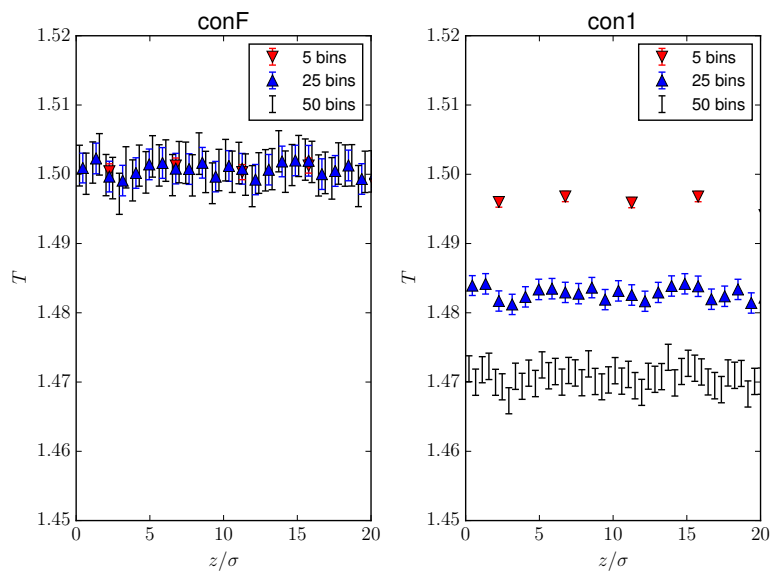

FIG. 1: Effect of number of subvolumes on the values of $T_{\text {con1 }}$

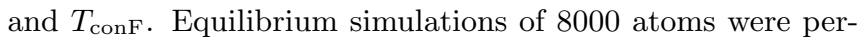
formed at $T=1.5, \rho=0.7$, with the system subdivided into between 5 and 100 slabs for the purposes of temperature calculation. While conF does not display any systematic deviation as the number of bins increases, the values of con1 decrease significantly as the number of bins increases (and thus as the number of particles contributing to the average decreases).

$T_{\text {conf }}$ and $T_{\text {con1 }}$ were computed using a varying number of bins, including bins with a characteristic width of the order of the one normally used in our NEMD simulations. We show in figure 1 the computations using all the definitions of temperature considered here for a system at $T=1.5$ and $\rho=0.7$ (representative of the "average" conditions of our NEMD simulations, see figures 3 and 7). It is clear that, unlike conF [28], con1 features a very strong dependence with the number of sub-volumes, becoming much less accurate as the number of bins increases. Increasing the number of bins results in a decrease in the number of particles per bin. By plotting the average temperature over all bins as a function of the number of bins in the system (figure 2), it can be seen that there is a dependence not only on the density but also on the total number of particles in each bin. This is a further consequence of the dyadic term which is neglected in arriving at equation (6), which introduces a discrepancy of the order $1 / N$, where $N$ is the number of atoms which contribute to the temperature measurement. When the simulation cell is divided into $N_{B}$ bins, the number of atoms in each bin is $N=\rho L_{x} L_{y} L_{z} / N_{B}$. As $N_{B}$ is varied in figure $2, N \sim 1 / N_{B}$ (the other terms remain constant at equilibrium), hence $T_{\text {con1 }} \sim N_{B}$. Despite its apparent similarity to $T_{\text {conF }}$, care must be taken when applying $T_{\text {con1 } 1}$. Even under conditions where its density dependence might be considered negligible, using it to evaluate local temperatures within subvolumes introduces an extra discrepancy.

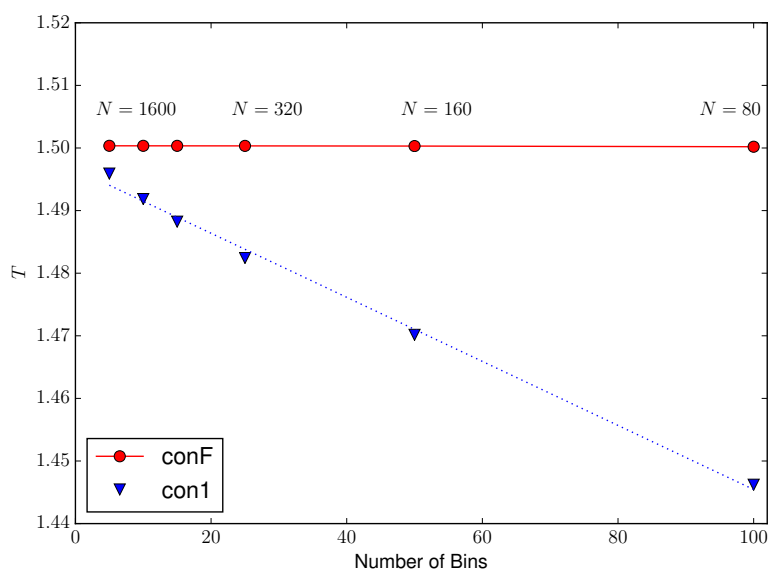

FIG. 2: "Binned" temperatures of figure 1 averaged over all bins and displayed as a function of number of bins. As explained in the text, this is equivalent to plotting against $1 / N$, where $N$ is the number of atoms which contribute to the temperature measurement. The text labels display the approximate number of atoms in each bin.

We now turn our attention to calculating thermal properties using configurational temperatures. We performed simulations of the Lennard-Jones fluid under a thermal gradient using the boundary-driven method discussed in section II. We obtained a fluid in a nonequilibrium steady state at average temperature $T=1.48$ and density $\rho=0.8$ along the $P=3.2$ isobar, using $T_{\mathrm{HOT}}=1.8$ and $T_{\mathrm{COLD}}=1.2$ for the hot and cold thermostatting layers. As noted in section II we thermostatted the boundary layers (shaded in figure 3) using a velocity rescale approach.

The resulting temperature profiles are displayed in figure 3. A single, monotonic, temperature profile was obtained by averaging the two thermal gradients around the center of mass of the box, exploiting the symmetry of the simulation cell. In accordance with previous work [28], we find that $T_{\text {conF }}$ is in excellent agreement with $T_{\text {kin }}$, even under the influence of a large temperature gradient $\left(|\nabla T|=0.06 \mathrm{~T} / \sigma, \sim 2 \times 10^{10} \mathrm{~K} / \mathrm{m}\right.$ in argon units). Following the bin volume dependence reported in figures 


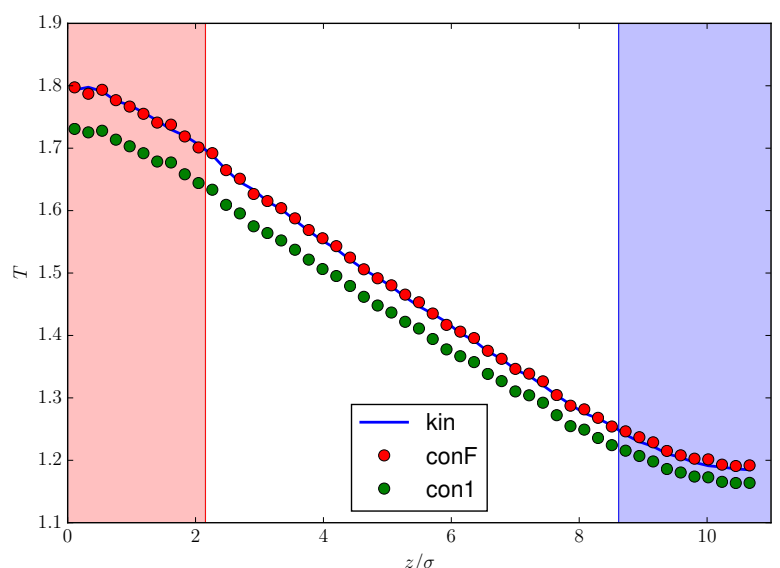

FIG. 3: Comparison of the three temperatures under an applied temperature gradient. The temperature gradient was imposed by thermostatting the atoms in the red (hot) and blue (cold) shaded regions using velocity rescaling. The temperature profile has been "folded" about the centre of the box, exploiting the symmetry in the $z$ direction. The errors in $T_{\text {conf }}$ and $T_{\text {con1 }}$ are of the same magnitude as the point size, while the errors in $T_{\text {kin }}$ are too small to display on this scale.

1 and 2 , the local calculation of $T_{\text {con1 }}$ causes it to underestimate the true temperature by around $2.5 \%$. We investigate in the following whether these temperature deviations translate into different thermal gradients, and consequently different thermal conductivities. To address this issue we computed the local thermal conductivity following reference [20], using both the configurational and kinetic thermal gradients. For the thermal conductivity computations we discarded the data in the thermostatted regions. Quadratic polynomials were then fitted to the temperature profiles in the interval $2.15<z<8.62$, i.e. excluding the thermostating regions, and the derivatives of the profiles were used to evaluate the local thermal gradients, $\mathrm{d} T(z) / \mathrm{d} z$, which were substituted into Fourier's law,

$$
J_{Q}=-\lambda(z) \frac{\mathrm{d} T(z)}{\mathrm{d} z}
$$

to yield the thermal conductivity, $\lambda$. The heat flux, $J_{Q}$, which is constant along the box (except in the thermostatting regions where it reverses signs to comply with the cell boundary conditions) is evaluated by invoking the conservation of energy, and the continuity equation, $J_{Q}=Q /(2 A \delta t)$, as discussed in section II. All the computations were performed by dividing the simulation box in bins perpendicular to the direction of the flux, with a bin width of $\sim 0.231 \sigma$.

The thermal conductivity results are displayed in figure 4 as a function of temperature. Our values are within the range of those reported in previous works [40]. The thermal conductivities reflect the differences between the temperatures reported above. $\lambda_{\text {kin }}$ and $\lambda_{\text {conF }}$ yield ex-

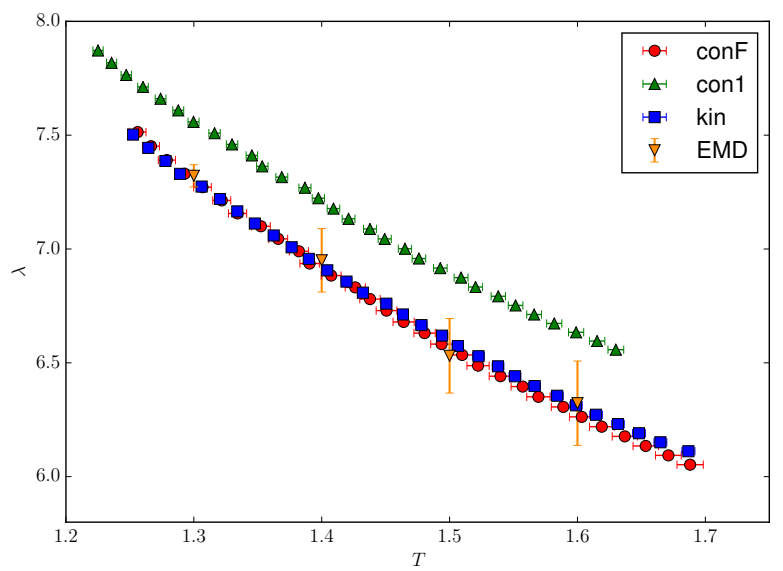

FIG. 4: Calculation of the thermal conductivity of the liquid system with an applied temperature gradient (figure 3). Thermal conductivities were determined by measuring the local gradient of the temperature profiles for each temperature expression (details in the text), and the heat flux $J_{Q}=0.463$. The points labelled "GK" indicate the results of equilibrium MD simulations on the same isobar as our NEMD results $(P=3.2)$. Where not displayed, error bars are of the same order as the point size.

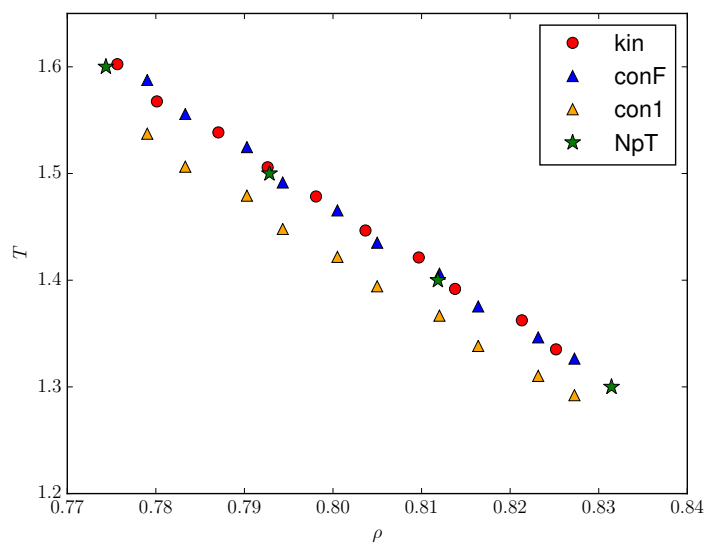

FIG. 5: Equations of state of the liquid system with an applied temperature gradient (figure 3). The points labelled "NpT" indicate the results of equilibrium MD simulations on the same isobar as our NEMD results $(P=3.2)$. Error bars are of the same order as the point size.

tremely similar results, while $\lambda_{\text {con1 }}$ is noticeably higher, with maximum deviations of about $5 \%$. This discrepancy is larger than the statistical uncertainty of our data, which is a maximum for the conF data where it is around $1 \%$. Since the flux is the same in all cases, the origin of the differences is entirely due to the approach used to compute the temperature gradients, and the inaccuracy of using the con1 equation to compute the temperature. We have also compared the NEMD results to those of equilibrium MD (EMD) simulations using the Green- 
Kubo relation,

$$
\begin{gathered}
\lambda=\frac{V}{3 k_{B} T^{2}} \int_{0}^{t_{\max }}\left\langle\mathbf{J}_{Q}(0) \cdot \mathbf{J}_{Q}(\tau)\right\rangle \mathrm{d} \tau \\
\mathbf{J}_{Q}=\frac{1}{V}\left\{\sum_{i}^{N}\left(m_{i} \mathbf{v}_{i}^{2}+\phi_{i}\right) \mathbf{v}_{i}+\frac{1}{2} \sum_{i, j}^{N}\left(\mathbf{f}_{i j} \cdot \mathbf{v}_{i}\right) \mathbf{r}_{i j}\right\}
\end{gathered}
$$

where in equation (10) (the Irving-Kirkwood heat flux [41]), $\phi_{i}$ denotes the potential energy of atom $i$ and the remaining terms take their conventional meanings, and $t_{\max }$ in equation (9) indicates the maximum integration time employed to calculate the GK integral. We performed simulations in the $\mathrm{NpT}$ ensemble for $10^{6}$ steps, using the Nosé-Hoover barostat and thermostat to bring the EMD simulations to the required pressure $(P=3.2)$ and temperature. The autocorrelation functions were then evaluated over $2 \times 10^{7}$ steps in the NVE ensemble, with values of $\mathbf{J}_{Q}$ sampled every step. The final value was determined by averaging over the plateau region of the integral in equation (9) up to $t_{\max }=5$ (2000 timesteps). The EMD results are found to agree well with the results obtained using the $T_{\text {conF }}$ and $T_{\text {kin }}$ values only, highlighting again the inadequacy of the con 1 definition for the computation of thermal conductivities.

The temperature and density pairs within each slab of the NEMD simulation can be used to construct the equation of state of the fluids along an isobar. We show in figure 5 the results for $P=3.2$, which corresponds to the pressure of our NEMD simulations. Equilibrium MD simulations were performed in the NpT ensemble at $P=3.2$ and four representative temperatures. The kin and conf temperatures clearly give rise to an equation of state that is fully consistent with the EMD results, while the con 1 results are shifted down.

\section{B. Interfaces}

We have shown above that the configurational $\left(T_{\mathrm{conF}}\right)$ and kinetic temperatures yield very similar thermal conductivities, and that the corresponding thermal gradients and equations of state are compatible with equilibrium Green-Kubo and NpT ensemble simulations, while the $T_{\text {con1 }}$ approximation is not suitable for these purposes. It has been shown recently [28] that $T_{\text {conF }}$ and $T_{\text {kin }}$ are in good agreement across a liquid-vapour interface. We extend this analysis by calculating the thermal conductance across the interface, and also consider a solid-liquid interface. The thermal conductance can be computed in a similar way to the thermal conductivity discussed above, namely by the analysis of the different thermal gradients across the interface.

We simulated a liquid-vapour interface at equilibrium conditions and computed the temperature profile across the system by dividing the simulation box into 100 bins of width $0.575 \sigma$. The liquid-vapour interface was constructed by taking an equilibrated liquid simulation cell ( $\rho=0.8, T=0.95$ ) and instantaneously increasing the length of the $\mathrm{z}$ direction to give an overall density of $\rho=0.3$. A simulation of $10^{5}$ timesteps in the NVT ensemble at $T=0.95$, followed by the same number of timesteps in the NVE ensemble, resulted in an equilibrated system with a liquid/vapour interface perpendicular to the long axis of the simulation cell. Production simulations of $2.5 \times 10^{6}$ steps were then performed, with data for temperature calculation recorded every 100th step. The final temperature profiles were achieved by averaging data over three independent simulations. We show in figure 6 the density and temperature profiles for a representative thermodynamic state at an intermediate temperature, $T \sim 0.95$. The $T_{\text {kin }}$ profile shows good convergence with, larger fluctuations in the vapour phase, as the average number of particles per bin is small, $\sim 25$ in the vapour bins compared to $\sim 760$ in the liquid bins.

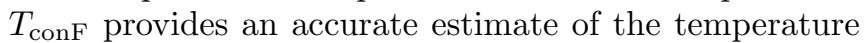
profile, though one which is considerably noisier than $T_{\text {kin }}$ in the vapour region, making difficult the evaluation of systematic deviations with respect to the kinetic approach. On the other hand, $T_{\text {con1 }}$ features important deviations from the kinetic temperature (see figure $6 \mathrm{~b}$ ). In the liquid phase, we find differences of the order of $0.3 \%$. This is smaller than the $\sim 1 \%$ discrepancy which might be expected by considering figure 2 , but we note that the density in the liquid region of our system is slightly higher, around $\rho=0.75$.

In the vapour phase, however, the temperature is underestimated by $47 \%$. The con 1 approach thus introduces an artificial interfacial thermal gradient, which arises from the the strong dependence of $T_{\text {con1 }}$ on the number of atoms in each subvolume of the system.

Following the equilibrium analysis, we imposed a heat flux to our liquid-vapour system, using the procedure described in section II. We show in figure 7 the resulting thermal gradient along with the fluid density profile. The local kinetic temperature is well defined in the vapour phase, and it features the characteristic "jump" slightly before reaching the liquid surface. This temperature jump, $\Delta T$ provides a direct route to compute the thermal conductance, $G$,

$$
J_{Q}=-G \Delta T
$$

where $\Delta T$ measures the temperature jump. The latter was estimated by firstly fitting the function

$$
\rho(z)=\frac{1}{2}\left(\rho_{L}+\rho_{V}\right)-\frac{1}{2}\left(\rho_{V}-\rho_{L}\right) \tanh \left\{\frac{2}{\delta}\left(z-z_{0}\right)\right\}
$$

to the interfacial density profile, where $\rho_{L}$ and $\rho_{V}$ are the bulk densities of the liquid and the vapour, respectively, $\delta$ is the thickness of the interface, and $z_{0}$ is the location of the Gibbs dividing surface [42]. We 

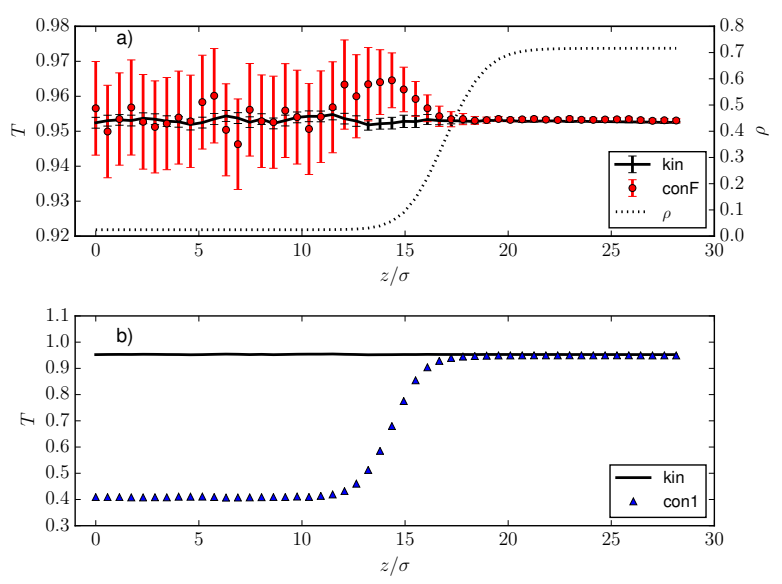

FIG. 6: Agreement between the various temperature measurements in a system containing a liquid/vapour interface at uniform temperature. a) $T_{\text {conF }}$ features maximum deviations of around $1 \%$. b) $T_{\operatorname{con} 1}$ agrees relatively well in the dense liquid region, but extremely poorly in the vapour. Where error bars are not shown, measurement errors are smaller than the size of the markers.
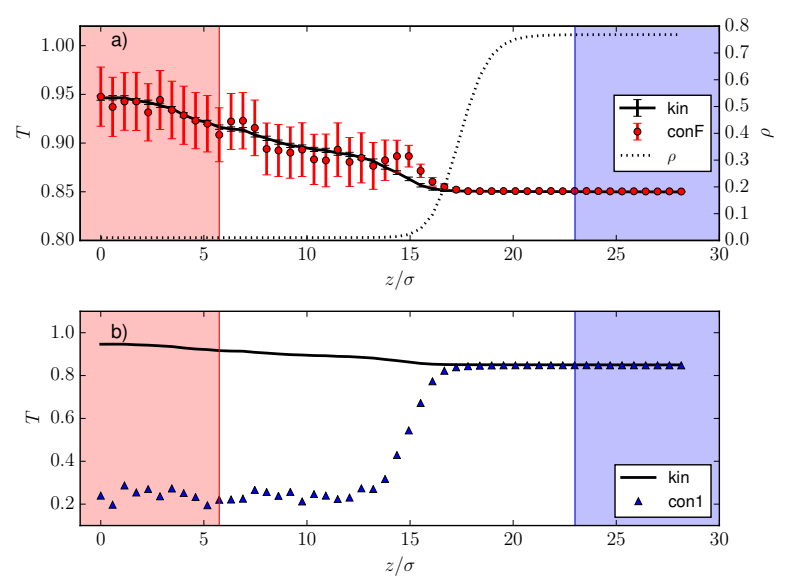

FIG. 7: Comparison of three temperature definitions for the same liquid/vapour interface system as in figure 6 , with an applied temperature gradient. The desired hot and cold temperatures, $T_{H}=0.95, T_{C}=0.85$, were enforced using velocity rescaling thermostats in the regions indicated by the red (hot) and blue (cold) shading, resulting in a heat flux of $J_{Q}=1.15 \times 10^{-3} \pm 4.56 \times 10^{-5}$. We have exploited the symmetry of the simulation cell to "fold" it about the centre, improving the statistics of the temperature computations.

then used a "1-99 rule" to divide the temperature profile into vapour and liquid sections: the vapour section of the temperature profile is that where the density is smaller than $1 \%$ of the bulk liquid value, and the liquid section that where the density is larger than $99 \%$ of the bulk liquid density. The temperature profiles in both the the liquid and vapour regions were fitted by straight lines which were then extrapolated into the in- terfacial region. Finally, the temperature jump was estimated by taking the difference between the extrapolated values at the Gibbs dividing surface, $z_{0}$, as indicated in figure 8 . The heat flux in the system was determined to be $J_{Q}=1.15 \times 10^{-3} \pm 4.56 \times 10^{-5}$. We obtain $G_{\text {kin }}=1.617 \times 10^{-1} \pm 2.750 \times 10^{-3}$. This thermal conductance is fairly low, $\sim 9 \mathrm{MW} /\left(\mathrm{m}^{2} \mathrm{~K}\right.$ ) (argon units) compared to biological interfaces [43], although this is not unexpected for this liquid-vapour interface [44, 45]. $T_{\text {conf }}$ features fairly strong fluctuations in the vapour phase, making the estimation of the temperature jump more difficult. The resulting thermal conductance is much lower than the one obtained from the kinetic route, $G_{\text {conF }}=2.549 \times 10^{-1} \pm 1.934 \times 10^{-2}$. Despite $T_{\text {conF }}$ agreeing within error with $T_{\text {kin }}$, the computed thermal conductance is amost $60 \%$ larger. We do not find this particularly surprising, in light of the much larger uncertainties in the $T_{\text {conF }}$ measurements. The configurational temperature route is less precise in the computation of thermal gradients in phases at low densities, as can be seen in the large fluctuations in computed temperatures (see figure 7).

We have represented our results for the temperature profiles using $T_{\text {con1 } 1}$ in figure $7 \mathrm{~b}$. As before, the approximate $T_{\text {con1 }}$ is shown to be inappropriate for these conditions - an artificial temperature jump is predicted at the interface that results in a negative thermal conductance, which is unphysical. $T_{\text {con1 }}$ is therefore not suitable for the computation of either interfacial thermal gradients or interfacial thermal transport coefficients.

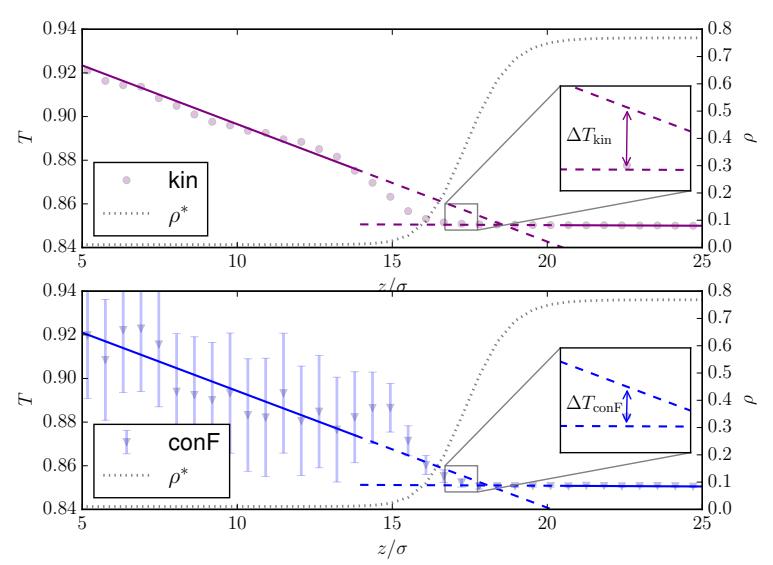

FIG. 8: Estimation of the temperature jump across the interface for the calculation of the interfacial thermal conductance. The density fitting procedure (see main text) yielded the parameters: $\rho_{L}=0.768, \rho_{V}=0.010, \delta=2.718$, with the Gibbs dividing surface at $z_{0}=17.44$. The lines are solid where they were fitted to the temperature profile, and dashed where they were extrapolated. The resulting temperature jumps are a) $\Delta T_{\text {kin }}=7.13 \times 10^{-3}$ b) $\Delta T_{\text {conF }}=4.52 \times 10^{-3}$.

Recent studies have focused on the computation of configurational temperatures in zeolite substrates [37], which provide a heterogeneous energetic environment to 
adsorbed fluids, e.g. argon. It was found that the configurational and kinetic temperatures were very different when the averages were computed in small sub-volume elements. The liquid-vapour interface discussed above provides a heterogeneous environment too. Despite this we find that the conF definition, which was also used in reference [37], provides a temperature profile in reasonable agreement with the kinetic one. To examine this question further we have considered a solid-liquid interface (see figure 9 for an example of the simulation setup) under a thermal gradient. In addition to the heterogeneous environment associated to the interface itself, the solid breaks the homogeneous structure characteristic of the liquid phase.

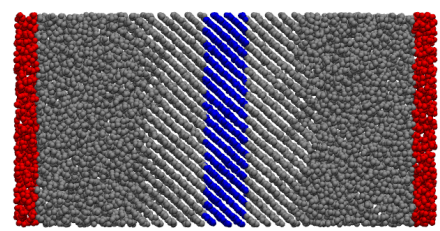

FIG. 9: Snapshot of the solid/liquid interface. The hot and cold thermostats were set to $T_{H}=1.8, T_{C}=1.2 \mathrm{using}$ velocity rescaling thermostats in the red (hot) and blue (cold) shaded regions.
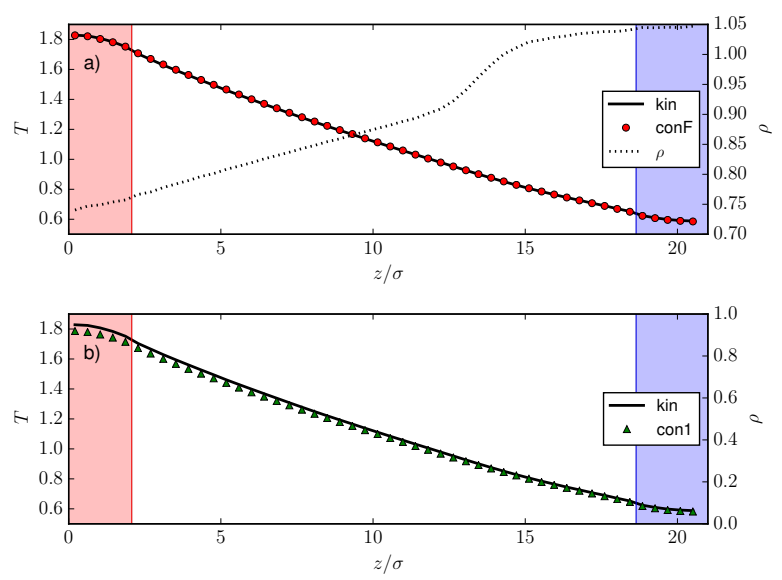

FIG. 10: Comparison of the various temperature calculations in a system with a solid/liquid interface (figure 9). (a) $T_{\text {conF }}$ and $T_{\text {kin }}$ are in almost exact agreement (errors are too small to represent). (b) $T_{\text {con1 }}$ agrees reasonably well with $T_{\text {kin }}$, with the same density dependent discrepancy seen in the other systems that we have considered.

We show in figure 10 the temperature profiles across the liquid-solid interface. $T_{\text {conF }}$ and $T_{\text {kin }}$ agree very well, with errors too small to display in figure 10. This is due to the larger number of atoms per slab in this higher density system $(\sim 150$ atoms per bin adjacent to the hot thermostat). Even the previously poor $T_{\text {con1 }}$ offers reasonable agreement with $T_{\text {kin }}$, with a maximum deviation (underestimation) of $\sim 1 \%$. We did not attempt the com- putation of the thermal conductance in the liquid-solid interface as the temperature jump is present is very small and an estimation with the method used above for the liquid-vapor interace would be imprecise. Recent works do indeed suggest that the temperature jump for the argon liquid-vapour interface is very small [46].

\section{CONCLUSIONS AND FINAL REMARKS}

The computation of the temperature in steady state systems is a topical problem of interest to theoreticians and experimentalists. Non-equilibrium simulations provide a route to investigate steady state systems and to quantify the transport properties of these systems. Configurational temperatures provide an independent route to quantify the temperature of non-equilibrium systems too. We have examined the applicability of two expressions for configurational temperatures to measure thermal gradients in non-equilibrium systems, taking into account the dependence of the configurational temperature with the size of the sub-volumes required to compute local temperatures and to construct temperature profiles. Our computations clearly show that the thermal gradient strongly depends on the definition of temperature employed. The temperatures and temperature gradients resulting from the conF expression are in excellent agreement with the kinetic temperature at thermodynamic states characteristic of dense fluids.

On the other hand, the approximate con1 expression can be extremely inaccurate. Under low density conditions, or when evaluated in subvolumes with a small number of atoms, it can result in temperature profiles that deviate from the kinetic one significantly $(\sim 60 \%$ in figure 6 ). The thermal gradient also deviates greatly, resulting in thermal conductivities that overestimate the ones obtained with the kinetic temperature or the conF configurational one by $5 \%$. Further, in the simulation of interfaces we find that $T_{\text {con1 }}$ predicts unphysical temperature jumps across the interface in equilibrium systems and in interfaces under thermal gradients, leading to unphysical negative thermal conductances.

We have further investigated the limitations of the configurational temperature in the computation of local temperatures in systems involving energetically heterogeneous environments. This question has been examined recently in the context of solid materials, where large deviations between the configurational and kinetic temperatures were reported when the size of the sub-volumes used to sample the temperature was small, on the order of a molecular diameter or less. The liquid-vapour and liquid-solid interfaces studied here, which involve also energetic and density inhomogeneities, provide a route to assess the accuracy of the configurational temperature in other systems. Letting aside the $T_{\text {con1 }}$ definition, which, as we have shown, is clearly wholly unsuited to the evaluation of temperature profiles when the number of atoms contributing to each point is smaller than a few thousand, 
we find that temperature profiles obtained from other configurational temperature expressions $\left(T_{\text {conF }}\right)$ are in close agreement with the kinetic temperature predictions. Hence, we do not find here evidence for a breakdown of the equivalence of configurational and kinetic temperatures when the temperature is computed in small subvolumes and inhomogeneous systems.

Previous non-equilibrium simulations have shown that the kinetic temperature provides consistent temperature profiles, in the sense that transport properties (namely the thermal conductivity) and the equation of the state of a system under a thermal gradient are fully consistent with the results obtained from equilibrium simulations. We have further validated this idea in the present paper. The kinetic temperature is in general simpler and better behaved than the configurational one [47], and less sensitive to local changes in the system. Indeed, configurational temperature definitions may contain spurious rotational contributions [48] and it has been suggested that it can be negative in far from equilibrium conditions, such as in the case of shock waves [49], where Yvon's theorem is no longer applicable. Overall, we have found no evidence to suggest systematic differences between the temperatures $T_{\text {conF }}$ and $T_{\text {kin }}$ under thermal gradients. That said, the kinetic temperature provides physically correct answers, is easy to implement and is more precise than the configurational temperature, and therefore represents an ideal approach for the computation of local temperatures, thermal gradients, and thermal transport properties.

\section{ACKNOWLEDGEMENTS}

This work was supported by the Engineering and Physical Sciences Research Council (EPSRC) (EP/J003859/1). The authors acknowledge the Imperial College High Performance Computing Service for providing computational resources. FB would like to thank the EPSRC for the award of a Leadership Fellowship. JMR would like thank the Leverhulme Trust for the award of a Visiting Professorship to Imperial College.

\section{Appendix A: Evaluating the temperature expressions}

Unlike the atomic momenta and forces, which are required for the dynamics simulation, the terms $\nabla_{i} \cdot \mathbf{F}_{i}$ (the divergences of the atomic forces) are not readily available in typical MD software packages.

For short ranged pair potentials, these terms can be evaluated using the following procedure:

$$
\begin{aligned}
\nabla_{i} \cdot \mathbf{F}_{i} & =\frac{\partial F_{i x}}{\partial r_{i x}}+\frac{\partial F_{i y}}{\partial r_{i y}}+\frac{\partial F_{i z}}{\partial r_{i z}}=\sum_{k}^{\{x, y, z\}} \frac{\partial F_{i k}}{\partial r_{i k}} \\
& =\sum_{k}^{\{x, y, z\}} \sum_{i \neq j} \frac{\partial}{\partial r_{i k}}\left(-\frac{f\left(r_{i j}\right)}{r_{i j}} r_{i j k}\right) \\
& =\sum_{k}^{\{x, y, z\}} \sum_{i \neq j}\left[\frac{f\left(r_{i j}\right)}{r_{i j}}-r_{i j k} \frac{\partial r_{i j}}{\partial r_{i k}} \frac{\partial}{\partial r_{i j}} \frac{f\left(r_{i j}\right)}{r_{i j}}\right] \\
& =\sum_{k} \sum_{i \neq j}\left[\frac{f\left(r_{i j}\right)}{r_{i j}}+\frac{r_{i j k}^{2}}{r_{i j}} \frac{\partial}{\partial r_{i j}} \frac{f\left(r_{i j}\right)}{r_{i j}}\right] \\
& =\sum_{i \neq j}\left[\frac{3 f\left(r_{i j}\right)}{r_{i j}}+r_{i j} \frac{\partial}{\partial r_{i j}} \frac{f\left(r_{i j}\right)}{r_{i j}}\right]
\end{aligned}
$$

For the Lennard-Jones potential (equation (7)),

$$
\begin{aligned}
\frac{f\left(r_{i j}\right)}{r_{i j}} & =\frac{24 \varepsilon_{i j}}{r_{i j}^{2}}\left[2\left(\frac{\sigma_{i j}}{r_{i j}}\right)^{12}-\left(\frac{\sigma_{i j}}{r_{i j}}\right)^{6}\right] \\
r_{i j} \frac{\partial}{\partial r_{i j}} \frac{f\left(r_{i j}\right)}{r_{i j}} & =\frac{96 \varepsilon_{i j}}{r_{i j}^{2}}\left[2\left(\frac{\sigma_{i j}}{r_{i j}}\right)^{6}-7\left(\frac{\sigma_{i j}}{r_{i j}}\right)^{12}\right] \\
\nabla_{i} \cdot \mathbf{F}_{i} & =\sum_{i \neq j} \frac{24 \varepsilon_{i j}}{r_{i j}^{2}}\left[5\left(\frac{\sigma_{i j}}{r_{i j}}\right)^{6}-22\left(\frac{\sigma_{i j}}{r_{i j}}\right)^{12}\right]
\end{aligned}
$$

948 (1987), ISSN 10502947.

[7] G. V. Paolini and G. Ciccotti, Physical Review A 35, 5156 (1987), ISSN 10502947.

[8] F. Bresme and B. Hafskjold, The Journal of Physical Chemistry 100, 1879 (1996), ISSN 0022-3654, URL http://pubs.acs.org/doi/abs/10.1021/jp9512321.

[9] A. Perronace, C. Leppla, F. Leroy, B. Rousseau, and S. Wiegand, Journal of Chemical Physics 116, 3718 (2002), ISSN 00219606.

[10] P. A. Artola and B. Rousseau, Physical Review Letters 98, 125901 (2007), ISSN 00319007.

[11] N. Galamba, C. A. Nieto de Castro, and J. F. Ely, The Journal of chemical physics 126, 204511 (2007), ISSN 0021-9606.

[12] M. Salanne, D. Marrocchelli, C. Merlet, N. Ohtori, and

[6] D. J. Evans and D. MacGowan, Physical Review A 36 , 
P. A. Madden, Journal of physics. Condensed matter : an Institute of Physics journal 23, 102101 (2011), ISSN 0953-8984

[13] J. Armstrong and F. Bresme, Physical chemistry chemical physics : PCCP 16, 12307 (2014), ISSN 1463-9084, URL http://www.ncbi.nlm.nih.gov/pubmed/24818599.

[14] F. Bresme, A. Lervik, and J. Armstrong, in Experimental Thermodynamics Volume X: Non-equilibrium Thermodynamics with Applications (The Royal Society of Chemistry, 2016), pp. 105-133, ISBN 978-1-78262-024-2.

[15] D. A. McQuarrie, Statistical Mechanics (Harper \& Row, New York, 1975).

[16] D. J. Evans and G. P. Morris, Statistical Mechanics of Nonequilibrium Liquids (Academic Press, San Diego, 1990).

[17] T. Ikeshoji and B. Hafskjold, Non-equilibrium molecular dynamics calculation of heat conduction in liquid and through liquid-gas interface (1994).

[18] F. Müller-Plathe, The Journal of Chemical Physics 106, 6082 (1997), ISSN 00219606, URL http://scitation.aip.org/content/aip/journal/ jcp/106/14/10.1063/1.473271.

[19] F. Römer, A. Lervik, and F. Bresme, Journal of Chemical Physics 137, 74503 (2012), ISSN 00219606.

[20] F. Bresme and J. Armstrong, The Journal of chemical physics 140, 016102 (2014), ISSN 1089-7690, URL http: //www.ncbi.nlm.nih.gov/pubmed/24410242.

[21] S. R. de Groot and P. Mazur, Non-Equilibrium Thermodynamics (Dover, New York, 1984).

[22] J. Casas-Vázquez and D. Jou, Temperature in nonequilibrium states: a review of open problems and current proposals (2003)

[23] P. J. Daivis and D. Jou, Continuum Mechanics and Thermodynamics 24, 37 (2012), ISSN 09351175.

[24] H. H. Rugh, Physical Review Letters 78, 772 (1997), ISSN 0031-9007, 9701026, URL http://link.aps.org/ doi/10.1103/PhysRevLett.78.772.

[25] J. P. Hansen and I. R. Macdonald, Theory of Simple Liquids, vol. 2 (Academic Press, 2013), ISBN 9780123705358

[26] L. D. Landau and E. M. Lifschitz, Statistical Physics, Part 1 (Pergamon, 1969), ISBN 978-0080230399.

[27] O. Jepps, G. Ayton, and D. Evans, Physical Review E 62, 4757 (2000), ISSN 1063-651X, URL http://link. aps.org/doi/10.1103/PhysRevE.62.4757.

[28] A. Lervik, Ø. Wilhelmsen, T. T. Trinh, and H. R. Nagel, The Journal of chemical physics 143, 114106 (2015), ISSN 1089-7690, URL http://www.ncbi.nlm.nih.gov/ pubmed/26395686.

[29] B. D. Butler, G. Ayton, O. G. Jepps, and D. J. Evans, Journal of Chemical Physics 109, 6519 (1998), ISSN 00219606

[30] D. G. Grier and Y. Han, J. Phys.: Condens Matter 16, 8 (2004), ISSN 0953-8984, 0404284, URL http://arxiv . org/abs/cond-mat/0404284.

[31] M. Mechelke and M. Habeck, Journal of Chemical Theory and Computation 9, 5685 (2013), ISSN 15499618, URL http://dx.doi.org/10.1021/ct400580p

[32] G. Rickayzen and D. M. Heyes, Journal of Chemical Physics 127, 144512 (2007), ISSN 00219606, URL http://scitation.aip.org/content/aip/journal/ jcp/127/14/10.1063/1.2793069.

[33] K. P. Travis and C. Braga, The Journal of Chemical Physics 128, 014111 (2008), ISSN 00219606, URL http://scitation.aip.org/content/aip/journal/ jcp/128/1/10.1063/1.2815768.

[34] P. J. Daivis, B. A. Dalton, and T. Morishita, Physical Review E 86, 056707 (2012), ISSN 1539-3755, URL http: //link.aps.org/doi/10.1103/PhysRevE.86.056707.

[35] G. Ayton, O. G. Jepps, and D. J. Evans, Molecular Physics 96, 915 (1999), ISSN 0026-8976, URL http://www . tandfonline.com/doi/abs/10.1080/ 00268979909483031.

[36] C. Baig and B. J. Edwards, Journal of Chemical Physics 132, 184906 (2010), ISSN 00219606.

[37] J. M. Simon and J. M. Rubi, Journal of Physical Chemistry B 115, 1422 (2011), ISSN 15206106.

[38] A. Baranyai, Temperature of nonequilibrium steady-state systems (2000).

[39] S. Plimpton, J. Comput. Phys. 117, 1 (1995), ISSN 00219991.

[40] M. Bugel and G. Galliero, Chemical Physics 352, 249 (2008), ISSN 03010104.

[41] J. H. Irving and J. G. Kirkwood, The Journal of Chemical Physics 18, 817 (1950), ISSN 00219606, URL http://link.aip.org/link/JCPSA6/ $\mathrm{v} 18 / \mathrm{i} 6 / \mathrm{p} 817 / \mathrm{s} 1\{\backslash \&\}$ Agg $=$ doi.

[42] F. P. Buff, R. A. Lovett, and F. H. Stillinger, Physical Review Letters 15, 621 (1965), ISSN 00319007, URL http://journals.aps.org/prl/abstract/ 10.1103/PhysRevLett.15.621.

[43] A. Lervik, F. Bresme, S. Kjelstrup, D. Bedeaux, and J. Miguel Rubi, Phys. Chem. Chem. Phys. 12, 1610 (2010).

[44] O. Wilhelmsen, D. Bedeaux, and S. Kjelstrup, Phys. Chem. Chem. Phys. 16, 10573 (2014).

[45] J.-M. Simon, S. Kjelstrup, D. Bedeaux, and B. Hafskjold, The Journal of Physical Chemistry B 108, 7186 (2004), ISSN 1520-6106, URL http://pubs.acs.org/doi/abs/ 10.1021/jp0375719.

[46] Z. Liang, W. J. Evans, and P. Keblinski, The Journal of Chemical Physics 141, 014706 (2014), ISSN 00219606, URL http://scitation.aip.org/content/aip/ journal/jcp/141/1/10.1063/1.4885849.

[47] W. G. Hoover and C. G. Hoover, Physical Review E - Statistical, Nonlinear, and Soft Matter Physics 77, 041104 (2008), ISSN 15393755, 0801.3051.

[48] W. G. Hoover and C. G. Hoover, Physical Review E - Statistical, Nonlinear, and Soft Matter Physics 80, 011128 (2009), ISSN 15393755, 0905.1913.

[49] W. G. Hoover and C. G. Hoover, Physical Review E - Statistical, Nonlinear, and Soft Matter Physics 79, 046705 (2009), ISSN 15393755, 0811.1807. 